\title{
Did dinosaurs have megakaryocytes? New ideas about platelets and their progenitors
}

\author{
Lawrence F. Brass
}

Departments of Medicine and Pharmacology, University of Pennsylvania, Philadelphia, Pennsylvania, USA.

\begin{abstract}
Biological evolution has struggled to produce mechanisms that can limit blood loss following injury. In humans and other mammals, control of blood loss (hemostasis) is achieved through a combination of plasma proteins, most of which are made in the liver, and platelets, anucleate blood cells that are produced in the bone marrow by megakaryocytes. Much has been learned about the underlying mechanisms, but much remains to be determined. The articles in this series review current ideas about the production of megakaryocytes from undifferentiated hematopoietic precursors, the steps by which megakaryocytes produce platelets, and the molecular mechanisms within platelets that make hemostasis possible. The underlying theme that connects the articles is the intense investigation of a complex system that keeps humans from bleeding to death, but at the same time exposes us to increased risk of thrombosis and vascular disease.
\end{abstract}

\section{Introduction}

Whether you accept post-Darwinian ideas about natural selection or prefer intelligent design, the systems that have evolved to limit blood loss following injury in humans and other mammals seem particularly well suited to the requirements of a closed circulatory system. In the relatively low-pressure, low-flow venous system, damage to the vascular wall exposes tissue factor, resulting in the activation of factor $\mathrm{X}$, the assembly of the prothrombinase complex, and the local production of the active form of the serine protease thrombin. Once activated, thrombin cleaves plasma fibrinogen to produce fibrin, which polymerizes to form the clot that arrests bleeding. In contrast, in the high-pressure, high-flow conditions found in the arterial system, thrombin and fibrin cannot accumulate fast enough to form a stable plug. Under these conditions, platelets are also required, in part to initiate formation of the hemostatic plug and in part to promote thrombin formation.

Blood platelets are anucleate cells that are produced in the bone marrow and then released into the circulation, where they remain for an average of 10 days. Eventually they are either consumed in thrombus formation or destroyed in the spleen. Circulating platelets have a characteristic discoid shape that originally gave rise to their name.

\section{Patching the vascular wall}

What sets platelets apart from other blood cells is their ability (while still inactive) to be captured by exposed collagen fibrils within an injured vessel wall, triggering a subsequent transformation in which the now-active platelets adhere tightly to the vessel wall and to each other (Figure 1). Activated platelets undergo a rapid cytoskeletal rearrangements, which allow them to spread on the exposed vascular wall, maximizing contact area at the expense of thickness. The spread platelets act as a base upon which additional platelets can accumulate, sticking to those that arrived first. In essence, platelets are circulating monitors of the integrity of the vascular wall. Once injury occurs and

Conflict of interest: The author has declared that no conflict of interest exists. Citation for this article: J. Clin. Invest. 115:3329-3331 (2005). doi:10.1172/JCI27111. platelets begin to accumulate, the growing mass of platelets not only serves as an obstacle to further bleeding but also promotes thrombin generation by supplying a 2-dimensional surface upon which coagulation factor $\mathrm{X}$-activating (tenase) and prothrombin-activating (prothrombinase) complexes can assemble and generate more thrombin, thereby enmeshing the platelets in a fibrin clot and helping to stabilize the hemostatic plug or thrombus. Activated platelets may also help to fuel the process of localized fibrin formation by capturing circulating microvesicles that contain additional tissue factor.

The evidence that platelets actually play the roles that were just described is drawn from histological examinations of arterial clots, studies on the activation of platelets in vitro, clinical observations of bleeding patients, and extensive work with animal models. As knowledge of platelet function has grown, so too has an appreciation of the contribution of platelets to ischemic cardiovascular and cerebrovascular disease, and the extent to which platelets are active participants in heart attacks and strokes. The cell surface receptors that allow platelets to be activated by collagen have been identified, as have the cell adhesion molecules, known as integrins, that allow one platelet to stick to another. Knowledge of normal platelet function has made possible the development of drugs that inhibit platelet activation by blocking cell surface receptors for platelet agonists, or that diminish intracellular signaling events and prevent the binding of platelets to each other. These drugs are in widespread clinical use. All of this suggests that hemostasis research is a closed book, the process fully explained.

\section{Unanswered questions}

What has become increasingly clear is that many of the most interesting aspects of platelet biology remain insufficiently understood. Newer methods that allow the direct observation of thrombus formation in vivo have raised as many questions as they have answered. What happens first at the site of injury? Is it thrombin generation or platelet adhesion? How do clotting and the formation of the platelet plug complement each other, and how does the platelet provide a regulated surface upon which clotting factor complexes can assemble? How is the rapid generation of sufficient thrombin promoted and then limited? 


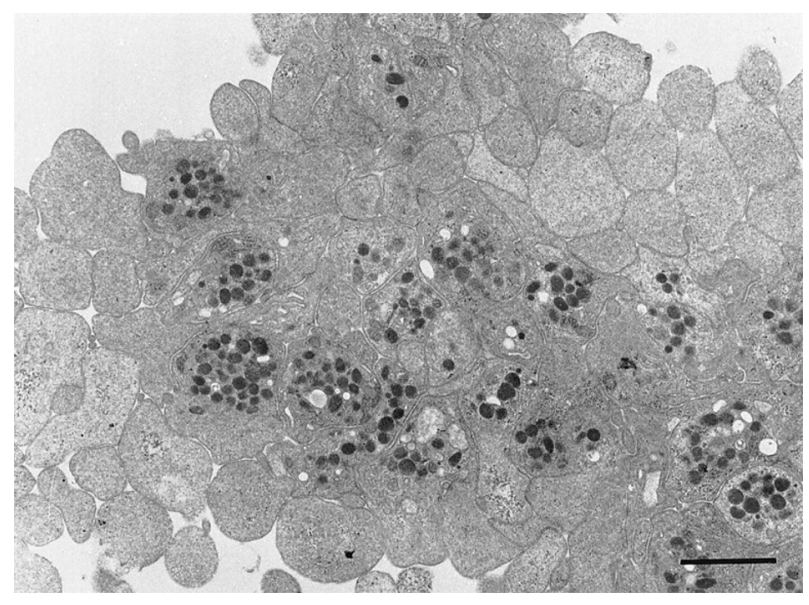

Figure 1

Platelets. Electron micrograph of human platelets activated by ADP. Scale bar: $1 \mu \mathrm{m}$. Reproduced with permission from Arteriosclerosis, Thrombosis, and Vascular Biology (16).

How are fast-moving, but not yet activated, platelets captured and retained? What keeps too many of them from accumulating, impeding blood flow and causing further tissue damage? What stabilizes the hemostatic plug and prevents it from falling apart before healing can occur? How are senescent platelets recognized, and why do platelets last only 10 days?

These questions are among the "hot topics" in the world of hemostasis research. At the same time, a separate, but related, line of investigation into the origin of platelets has opened. As already mentioned, platelets, like erythrocytes (red blood cells), lack a nucleus. However, the progression of platelets to an anucleate state occurs by a path that is entirely different from that of red cells. Erythrocytes begin as nucleated precursors that undergo gradual maturation within the bone marrow until, losing their nucleus, they enter the circulation as a carrier of hemoglobin. Platelets, on the other hand, arise from giant precursor cells (megakaryocytes) that reside and mature within the bone marrow, undergoing eventual cellular dissolution as they give rise to hundreds of individual platelets (Figure 2).

Until recently, little was known about the birth of platelets, but newer technologies that make it possible to observe megakaryocyte maturation and platelet formation ex vivo have started to provide new answers to old questions: How are platelets formed and released into the circulation? How are hematopoietic stem cells differentiated into megakaryocyte precursors, and how do those precursors mature into platelet-producing megakaryocytes? What regulates the pace of these events in vivo, and how might regulation of that process be manipulated in people with too many or too few platelets? How might differences in the delivery of megakaryocyte proteins into newly forming platelets result in differences among individuals in susceptibility to disease and responsiveness to drugs?

\section{In this series}

These questions and others are addressed by the authors of the articles that constitute this special Review series on platelet and megakaryocyte biology. The focus is on issues that are central to the understanding of platelet and megakaryocyte biology, but still in a state of ongoing investigation. In the first article of the series, Liyan
Pang, Mitchell Weiss, and Mortimer Poncz describe the transcriptional events that control megakaryocyte development and consider what happens when it goes wrong (1). Kenneth Kaushansky, whose studies helped to define the field, discusses how megakaryocyte maturation is driven by thrombopoietin and other growth factors (2). Sunita Patel, John Hartwig, and Joseph Italiano summarize and update their elegant studies on the birth of platelets from megakaryocytes (3). Bruce and Barbara Furie, pioneers in the development of new methodologies for studying thrombus formation in vivo, discuss the observations that they and others have made that have brought new insights to hemostasis research (4). Joel Bennett, who was one of the first to identify $\alpha_{\mathrm{II}} \beta_{3}$ as the platelet cohesion receptor, reviews recent developments in the understanding of how the integrin is activated in platelets (5). Nicholas Watkins and his colleagues share the particular frustrations and joys of applying proteomics technologies to questions about platelets and point out some of the directions in which those technologies might lead (6). Finally, Andreas May, Harald Langer, and Meinrad Gawaz discuss the studies that they and others have performed in order to understand how platelets contribute to thrombotic and inflammatory vascular disease (7), and Timothy Stalker, Li Zhu, and I consider the molecular interactions that help to maintain platelets in an active state and foster thrombus development (8).

\section{Hemostasis and dinosaurs}

This brings us back to the title of this essay. How do dinosaurs enter into a series of reviews about platelets and megakaryocytes? In Bones of the Earth, Michael Swanwick proposed that dinosaurs became extinct in part because of a failure of interspecies cooperativity after the collision of an asteroid with the Earth (9). To propose that the extinction of dinosaurs was instead a massive failure of hemostasis seems a bit over the top (and equally difficult to prove), but it raises fundamental questions about biological evolution. Not all species with a closed circulatory system have bone marrow megakaryocytes and circulating, anucleate platelets. Horseshoe crabs have a circulating nucleated cell that serves as both phagocyte and platelet (10). Fish have thrombocytes, nucleated cells with platelet-like properties $(11,12)$. Birds also have nucleated platelets $(13,14)$. Dinosaurs are no longer among us, but birds may be their descendants (15). Dinosaurs roamed the Earth far longer than humans have and arguably led a far more violent existence. Did dinosaurs have megakaryocytes? If dinosaurs had thrombocytes instead of platelets, did they require megakaryocytes? If not, why did a more complicated system arise in mammals?

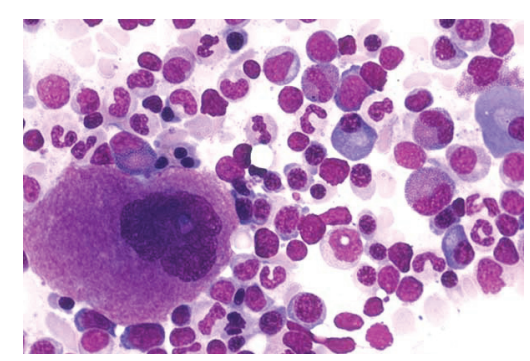

\section{Figure 2}

Megakaryocytes. Stained smear of cells from human bone marrow. The large cell in the lower left is a normal megakaryocyte. Magnification, $\times 50$. Reproduced with permission from Carden Jennings Publishing Co. (17). 
What survival and reproductive advantages are conferred by having platelets instead of thrombocytes? How many successful mutations did it take for thrombocytes to evolve into megakaryocytes and for megakaryocytes to be taught to make platelets, if that is indeed what happened? Stay tuned.

1. Pang, L., Weiss, M.J., and Poncz, M. 2005. Megakaryocyte biology and related disorders. J. Clin Invest. 115:3332-3338. doi:10.1172/JCI26720.

2. Kaushansky, K. 2005. The molecular mechanisms that control thrombopoiesis. J. Clin. Invest. 115:3339-3347. doi:10.1172/JCI26674.

3. Patel, S.R., Hartwig, J.H., and Italiano, J.E., Jr. 2005. The biogenesis of platelets from megakaryocyte proplatelets. J. Clin. Invest. 115:3348-3354. doi:10.1172/JCI26891.

4. Furie, B., and Furie, B.C. 2005. Thrombus formation in vivo. J. Clin. Invest. 115:3355-3362. doi:10.1172/JCI26987.

5. Bennett, J.S. 2005. Structure and function of the platelet integrin $\alpha_{\mathrm{IIb}} \beta_{3}$. J. Clin. Invest. 115:3363-3369. doi:10.1172/JCI26989.

6. Macaulay, I.C., et al. 2005. Platelet genomics and proteomics in human health and disease. J. Clin. Invest. 115:3370-3377. doi:10.1172/JCI26885.
Address correspondence to: Lawrence F. Brass, Departments of Medicine and Pharmacology, University of Pennsylvania, Room 915 Biomedical Research Building II, 421 Curie Boulevard, Philadelphia, Pennsylvania 19104, USA. Phone: (215) 573-3540; Fax: (215) 573-2189; E-mail: brass@mail.med.upenn.edu.
7. Gawaz, M., Langer, H., and May, A.E. 2005. Platelets in inflammation and atherogenesis. J. Clin. Invest. 115:3378-3384. doi:10.1172/JCI27196.

8. Brass, L.F., Zhu, L., and Stalker, T.J. 2005. Minding the gaps to promote thrombus growth and stability. J. Clin. Invest. 115:3385-3392. doi:10.1172/ JCI26869.

9. Swanwick, M. 2003. Bones of the Earth. HarperTorch. New York, New York, USA. 400 pp.

10. Levin, J. 1988. The horseshoe crab: a model for gram-negative sepsis in marine organisms and humans. Prog. Clin. Biol. Res. 272:3-15.

11. Thattaliyath, B., Cykowski, M., and Jagadeeswaran, P. 2005. Young thrombocytes initiate the formation of arterial thrombi in zebrafish. Blood. 106:118-124.

12. Jagadeeswaran, P., Gregory, M., Day, K., Cykowski, M., and Thattaliyath, B. 2005. Zebrafish: a genetic model for hemostasis and thrombosis. J. Thromb.
Haemost. 3:46-53.

13. Lacoste-Eleaume, A.S., et al. 1994. Biochemical and functional characterization of an avian homolog of the integrin GPIIb-IIIa present on chicken thrombocytes. Exp. Cell Res. 213:198-209.

14. Bertram, E.M., Jilbert, A.R., and Kotlarski, I. 1998. Characterisation of duck thrombocytes. Res. Vet. Sci. 64:267-270.

15. Zhou, Z. 2004. The origin and early evolution of birds: discoveries, disputes, and perspectives from fossil evidence. Naturwissenschaften. 91:455-471.

16. Humbert, M., et al. 1996. Ultrastructural studies of platelet aggregates from human subjects receiving clopidogrel and from a patient with an inherited defect of an ADP-dependent pathway of platelet activation. Arterioscler. Thromb. Vasc. Biol. 16:1532-1543.

17. BloodLine: the online resource for hematology education and news. http://image.bloodline.net/ stories/storyReader $\$ 1746$. 